\title{
Anthracene-Based Medium Bandgap Conjugated Polymers for High Performance Polymer Solar Cells Exceeding 8\% PCE Without Additive and Annealing Process
}

\author{
Jae Woong Jung, Feng Liu, Thomas P. Russell, and Won Ho Jo*
}

Rapid development of polymer solar cells (PSCs) based on bulk heterojunction structure composed of a conjugated polymer and a fullerene derivative as an electron-donor and acceptor, respectively, has led to dramatic advance in the solar cell performance. ${ }^{[1]}$ The power conversion effciency (PCE) of PSCs has significantly been increased over the last few years exceeding $10 \%$ mainly due to remarkable development of novel p-type conjugated polymers. ${ }^{[2]}$ Recent researches on the development of conjugated polymers have extensively focused on design and synthesis of low bandgap polymers in order to extend the light absorption range over $800 \mathrm{~nm}$ for enhanced photon absorption and thereby improved short-circuit current density $\left(U_{\mathrm{SC}}\right)$ of PSCs. ${ }^{[3]}$ However, low bandgap polymers are generally reported to exhibit high-lying highest occupied molecular orbital (HOMO) energy level due to reduced bandgap $\left(E_{\mathrm{g}}\right)$, which imposes a limitation on PSCs to achieve high open-circuit voltage $\left(V_{\mathrm{OC}}\right)$ and thus high PCE. ${ }^{[4]}$ Furthermore, the intrinsic narrow absorption range of low bandgap polymer, which significantly limits the use of full solar spectrum, is far from the ideal broad photon absorption. ${ }^{[5]}$

In this regard, medium bandgap polymers $\left(E_{\mathrm{g}}>1.7 \mathrm{eV}\right)$ with superior photovoltaic properties are highly desirable for high performance PSCs. Although the light absorption of medium bandgap polymer is limited in relatively short wavelength range, the morphology control and device engineering may afford improved exciton generation and charge transport for high $J_{\mathrm{SC}}$, which is comparable to the PSCs based on low bandgap polymers. ${ }^{[6]}$ In conjunction with their intrinsic lowlying HOMO energy levels which assure higher $V_{\mathrm{OC}}$ 's, the photovoltaic performance of medium bandgap polymer has been improved over $7 \%$ PCE. $^{[7]}$

One of the most challenging aspects in designing medium bandgap polymer is to properly choose electron-donating and electron-withdrawing building blocks for constructing donoracceptor (D-A)-type copolymers. The (D-A)-type copolymers are expected to exhibit medium bandgap, optimal energy levels, coplanar molecular structure as well as good charge transport

Dr. J. W. Jung, Prof. W. H. Jo

Department of Materials Science and Engineering Seoul National University

1G wanak-ro, Gwanak-gu, Seoul 151-744, SouthKo rea E-mail:whjpoly@snu.ac.kr

Dr. F. Liu, Prof. T. P. Russell

Department of Polymer Science and Engineering

University of Massachusetts

Amherst, MA 01003, USA

DOI: 10.1002/aenm.201500065 characteristics. ${ }^{[8]}$ Moreover, the molecular frontier orbitals of (D-A)-type copolymer can be readily tuned by rational choice of $\mathrm{D}$ and $\mathrm{A}$ units. More specifically, it has been recognized that the electron-donating and electron-withdrawing powers of $\mathrm{D}$ and $\mathrm{A}$ units should be weak for obtaining medium bandgap copolymer. ${ }^{[9]}$ Particularly, weak electron-donating characteristic of D unit ensures a low-lying HOMO energy level of the resulting conjugated polymer, which is beneficial to achievement of high $V_{\mathrm{OC}}$ of PSCs.

Among many building blocks for D unit, anthracene has recently been used as the electron-donating moiety to construct (D-A)-type copolymers for PSCs. ${ }^{[10]}$ Owing to its weak electrondonating characteristic, anthracene-based polymers possess deep HOMO energy level, which is favorable for high $V_{\mathrm{OC}}$ in PCSs. Moreover, the planar and rigid nature of anthracene unit affords the corresponding copolymer to exhibit high charge transporting properties. ${ }^{[11]}$ In addition, the functionalization of anthracene through its 9,10-positions offers a viable way to finetune the optoelectronic properties with high solution processability of the corresponding polymer. ${ }^{[12]}$ Therefore, anthracene derivatives are very promising candidate as relatively weak D unit for construction of medium bandgap copolymer for high performance PSCs.

In this communication, we designed and synthesized medium bandgap (D-A)-type copolymers, comprising 9,10-thienylanthracene (TA) and benzothiadiazole (BT) as D and A unit, respectively. To optimize the molecular structure of the copolymer for high photovoltaic performance, four BT derivatives with different substituents were utilized for constructing the D-A copolymers. All four copolymers displayed low-lying HOMO energy levels and medium bandgaps in the range of (1.8-2.1) eV. Among them, fluorine-substituted polymers exhibited high crystallinity, which facilitates efficient chargecarrier transport and thus high $J_{\mathrm{SC}}$. Also, the fluorinated polymers showed superior photo-induced charge generation with suppressed bimolecular recombination, which is beneficial to achievement of high $J_{\mathrm{SC}}$. More importantly, the fluorinated polymers formed nanoscale phase-separated bulk heterojunction morphology without addition of processing additive and post treatment, leading to a promising PCE as high as $8.05 \%$, which is among the best value reported for medium bandgap polymers. This work clearly demonstrates that TA and fluorinated BT units are very promising D and A units for medium bandgap copolymer for high photovoltaic performance.

The copolymers composed of TA and BT units are synthesized by Stille cross-coupling polymerization in toluene with a Pd catalyst, as shown in Scheme 1. When the number average molecular weights $\left(M_{n}\right)$ and polydispersity indexes (PDI) of 

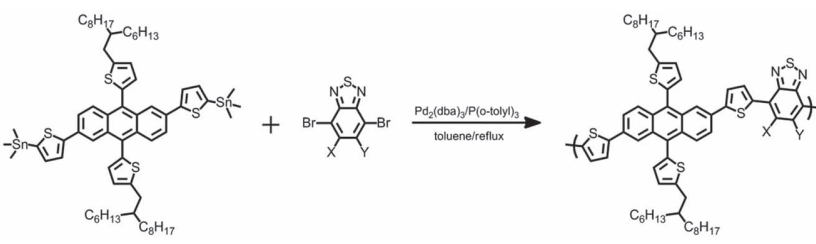

PTADTBT $(X, Y=H)$

PTADTFBT $(X=F, Y=H)$

PTADTDFBT $(X=F, Y=F)$ PTADTBTO $\left(X, Y=O_{8} \mathrm{H}_{17}\right)$

Scheme 1. Synthesis of medium bandgap polymers studied in this work.

polymers are measured by gel permeation chromatography (GPC), it reveals that all four polymers have sufficient molecular weight $(\approx 40 \mathrm{kDa})$ for device application (Table 1). All polymers have high solubility in chlorinated organic solvents such as chloroform, chlorobenzene, and o-dichlorobenzene at room temperature, and also exhibit high thermal stability with $5 \%$ weight loss at $350{ }^{\circ} \mathrm{C}$ (see Supporting Information). The optical properties of the copolymers were measured by an UV-vis absorption spectrometer and the relevant data are summarized in Table 1 . In solution state, the copolymer without any substituent on BT (PTADTBT) exhibits broad absorption in the range of 400-700 $\mathrm{nm}$ with an absorption onset at $695 \mathrm{~nm}$ corresponding to an optical bandgap $\left(E_{\mathrm{g}}\right)$ of $1.78 \mathrm{eV}$. As the fluorine atoms are introduced at the $\mathrm{BT}$ unit, the absorptions of the polymers are slightly blue-shifted, and thus the $E_{\mathrm{g}}$ 's of fluorinated polymers (PTADTFBT and PTADTDFBT) are increased to 1.80 and $1.91 \mathrm{eV}$, respectively. The widened bandgaps of fluorinated polymers can be attributed to the inductive electron-withdrawing effect of fluorine atom on the aromatic ring of the conjugated polymer backbone. ${ }^{[13]}$ However, PTADTBTO with alkoxy side group as a substituent exhibits a large blueshift in absorption with the highest $E_{\mathrm{g}}$ of $2.10 \mathrm{eV}$. The high $E_{\mathrm{g}}$ of the alkoxy-substituted polymer may arise from less electrondeficient nature of alkoxy-substituted BT than other BT derivatives, because strong electron-donating property of alkoxy group reduces the electron-withdrawing capability of $\mathrm{BT}$ unit, raising the LUMO energy level of PTADTBTO. It is worth pointing out that PTADTBT, PTADTFBT and PTADTDFBT show distinct vibronic shoulder peaks in solution while PTADTBTO does not exhibits a vibronic shoulder, indicating that the polymer chains except for PTADTBTO are partially ordered in solution due to strong intermolecular interaction (Figure 1a). In film state, all polymers exhibit pronounced bathochromic shift and broadening of absorption band extending to long wavelengths, as shown in Figure $1 b$, indicating that the polymer chains are effectively self-assembled owing to strong intermolecular $\pi-\pi$ interaction. ${ }^{[14]}$ Consequently, the formation of well-developed polymer crystallites is expected, which is beneficial to efficient charge transport and thus high photocurrent density.
When electrochemical properties of polymer films are measured by cyclic voltammetry, as shown in Figure 1c, PTADTBT, PTADTFBT, PTADTDFBT, and PTADTBTO have the HOMO energy levels of $-5.40,-5.48$, -5.55 , and $-5.38 \mathrm{eV}$, respectively, indicating that the fluorine atom substitution effectively lowers the HOMO energy level while the alkoxy substitution does not affect significantly the HOMO energy level of the copolymer. The LUMO energy levels of fluorinated polymers (PTADTFBT and PTADTDFBT) are slightly lower than nonfluorinated one (PTADTBT), while PTADTBTO exhibits very high-lying LUMO energy level of $-3.28 \mathrm{eV}$ because of less electron-deficiency of alkoxy-substituted BT, as discussed above. The effect of different substituents on the electrochemical characteristics of conjugated polymers can also be assessed from the molecular orbital distribution at HOMO and LUMO energy levels calculated from the density functional theory (DFT). As depicted in Figure S1 (Supporting Information), the HOMO electrons are distributed mainly on electron-rich TA unit (extending slightly onto the thiophene unit), whereas the LUMO electrons are localized on electron-deficient BT unit for PTADTBT, PTADTFBT, and PTADTDFBT. However, PTADTBTO shows featureless distribution of HOMO and LUMO electrons due to reduced electron-deficiency of BT unit by strongly electron-donating alkoxy groups. It should be also noted here that the LUMO electrons of PTADTDFBT are delocalized over electrondonating TA unit, indicating that quinoidal structure (strong donor-acceptor hybridization) in PTADTDFBT is more likely expected than other polymers. ${ }^{[15]}$

The structural information such as crystallinity, molecular orientation, intermolecular distance, and crystallite size of the polymers in thin-film state was obtained from the grazing incidence wide angle X-ray scattering (GIWAXS), as shown in Figure 2. PTADTBT shows strong (100) and (200) diffractions in the outof-plane direction $\left(q_{z}\right)$, indicating that most of crystallites have edge-on orientation. As fluorine atoms are substituted, the (100) and (200) diffractions in $q_{z}$ direction become stronger with clear observation of (300) and (400) peaks, indicating that fluorinated polymers form highly crystalline and dense crystallites. However, the polymer (PTADTBTO) with alkoxy group substitution shows weak and arc (100) diffraction, indicating that the polymer has low crystallinity with random orientation of crystallites. Although the edge-on orientation of polymer crystals would not be favorable for charge transport in the PSC device, an increase in the overall crystallinity of conjugated polymer is very beneficial to efficient charge transport and suppressed recombination of free charge carriers in PSCs. The effect of substituent on polymer

Table 1. Characteristics of the polymers synthesized in this work.

\begin{tabular}{|c|c|c|c|c|c|c|c|c|c|c|}
\hline Polymer & $\begin{array}{c}M_{\mathrm{n}} \\
{[\mathrm{kDa}]}\end{array}$ & PDI & $\begin{array}{c}\lambda_{\max , \mathrm{s}} \\
{[\mathrm{nm}]}\end{array}$ & $\begin{array}{c}\lambda_{\text {onset,s }} \\
{[\mathrm{nm}]}\end{array}$ & $\begin{array}{l}\lambda_{\max , \mathrm{f}} \\
{[\mathrm{nm}]}\end{array}$ & $\begin{array}{c}\lambda_{\text {onset, } \mathrm{f}} \\
{[\mathrm{nm}]}\end{array}$ & $\begin{array}{l}\text { LUMO } \\
{[\mathrm{eV}]^{\mathrm{a})}}\end{array}$ & $\begin{array}{c}\text { HOMO } \\
{[\mathrm{eV}]^{\mathrm{b})}}\end{array}$ & $\begin{array}{c}E_{\mathrm{g}} \\
{[\mathrm{eV}]^{\mathrm{c})}}\end{array}$ & $\begin{array}{l}\Delta \mu_{\mathrm{ge}} \\
{[\mathrm{D}]^{\mathrm{d})}}\end{array}$ \\
\hline PTADTBT & 41.1 & 1.59 & 574 & 695 & 650 & 710 & -3.62 & -5.40 & 1.78 & 9.51 \\
\hline PTADTFBT & 35.3 & 1.72 & 555 & 690 & 640 & 700 & -3.68 & -5.48 & 1.80 & 11.99 \\
\hline PTADTDFBT & 38.5 & 1.96 & 562 & 650 & 622 & 675 & -3.64 & -5.55 & 1.91 & 23.29 \\
\hline PTADTBTO & 40.4 & 1.98 & 521 & 590 & 582 & 645 & -3.28 & -5.38 & 2.10 & 11.61 \\
\hline
\end{tabular}

${ }^{\text {a) }} E_{\mathrm{g}}+\mathrm{HOMO}$; ${ }^{\mathrm{b})}$ Determined from cyclic voltammetry; ${ }^{\mathrm{c})}$ Determined from the onset of UV-vis absorption spectra; ${ }^{\mathrm{d})} \Delta \mu_{\mathrm{ge}}=\left[\left(\mu_{\mathrm{gx}}-\mu_{\mathrm{ex}}\right)^{2}+\left(\mu_{\mathrm{gy}}-\mu_{\mathrm{ey}}\right)^{2}+\left(\mu_{\mathrm{gz}}-\mu_{\mathrm{ez}}\right)^{2}\right]^{1 / 2}$ 

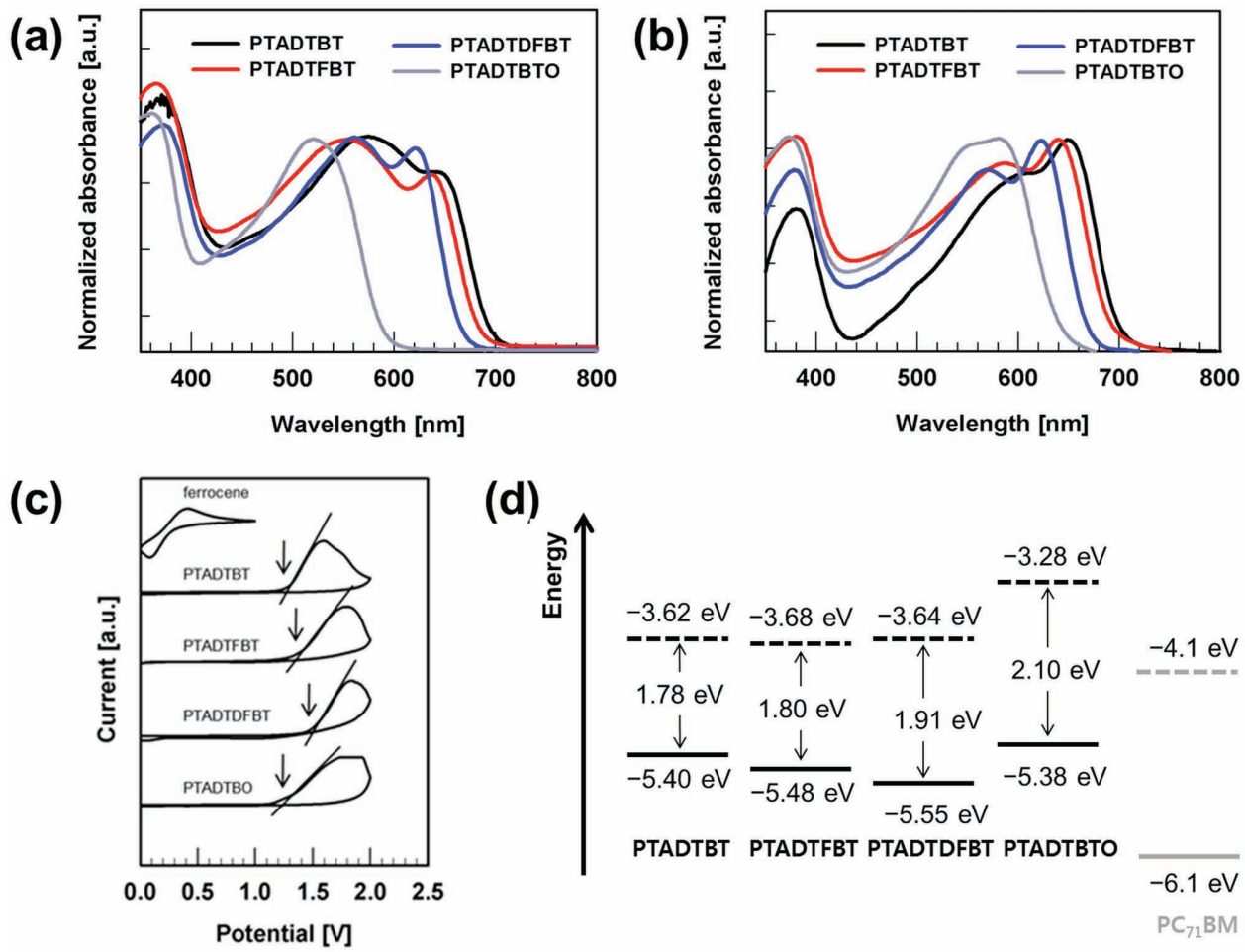

Figure 1. a) UV-vis absorption spectra in solution state; b) film state; c) cyclic voltammograms; and d) energy level diagram of the copolymers based on TA and BT derivatives.

crystallinity was further supported by the calculated torsional angle and planarity of the optimized geometry of the polymer backbones (Figure S2, Supporting Information). The 2-hexyldecyl side groups of the model compounds were replaced to short ethyl groups for DFT calculation to save the computation time in this work, although long and bulky side groups may induce distortion of the polymer backbone. ${ }^{[15 b]}$ The planarity of the polymer chain is increased as fluorine atom is introduced on BT unit (PTADTBT < PTADTFBT < PTADTDFBT), while PTADTBTO shows the most distorted structure with the largest torsional angles (Table S1, Supporting Information). This is because fluorine atom does not significantly cause steric hindrance owing to small atomic size (van der Waals radius, $r=1.35 \AA$ ) while long alkoxy group may cause strong steric hindrance. The crystallinity of polymer is closely related to the charge transport of conjugated polymer: Both the field effect transistor (FET) and the space charge limited current (SCLC) hole mobilities increase in the order of PTADTBTO < PTADTBT $<$ PTADTFBT $<$ PTADTDFBT (see Figures S3,S6; and also see Table S1, Supporting Information), which is very consistent with the crystallinity. It should be noted here that high crystallinity is advantageous for improved charge transport of the conjugated polymer, but too strong selfaggregation of high crystalline polymer may form large-sized crystal domain, which hinders exciton dissociation. Hence, the balance between crystallinity and domain size should be considered for high efficiency of PSCs.

The photovoltaic performances of the polymers were measured with an inverted device architecture. When the blend ratio of polymer to $\mathrm{PC}_{71} \mathrm{BM}$ was varied for optimization of the photovoltaic performance of PSCs, it was found that the optimum ratio was 1:3 for PTADTBT, PTADTFBT, and PTADTDFBT, while PTADTBTO showed the best result at the ratio of 1:4 (see Table S2, Supporting Information). Figure 3a shows the current density-voltage $(J-V)$ characteristics of optimized PSCs, and relevant photovoltaic properties are listed in Table 2. The PSC device based on PTADTBT exhibits a promising PCE of $6.92 \%$ with $V_{\mathrm{OC}}$ of $0.92 \mathrm{~V}, J_{\mathrm{SC}}$ of $11.61 \mathrm{~mA} \mathrm{~cm}^{-2}$ and FF of 0.65 . The substitution of one fluorine atom on BT unit (PTADTFBT) leads to enhanced PCE exceeding 7\%, which arises mainly from largely increased $V_{\mathrm{OC}}$ of $0.95 \mathrm{~V}$ due to lower-lying $\mathrm{HOMO}$ energy level. When two fluorine atoms are substituted on BT unit (PTADTDFBT), $V_{\mathrm{OC}}$ was further increased up to $0.97 \mathrm{~V}$, which is one of the highest $V_{\mathrm{OC}} \mathrm{S}$ of medium-bandgap polymers reported so far, and $J_{\mathrm{SC}}$ was also increased to $12.77 \mathrm{~mA} \mathrm{~cm}$. As a consequence, the PCE of PTADTDFBT increases as high as $8.05 \%$. However, the PCE of PTADTBTO was dropped to $4.64 \%$, mainly due to decreased $V_{\mathrm{OC}}$ and $J_{\mathrm{SC}}$, which are attributed to high-lying HOMO level and wide bandgap, respectively.

To understand the variation of $J_{\mathrm{SC}}$ of the device with different substitution, the maximum exciton generation rate $\left(G_{\max }\right)$ of device is estimated by plotting the photocurrent density $\left(J_{\mathrm{ph}}\right)$ as a function of effective voltage $\left(V_{\text {eff }}\left(J_{\text {ph }}=J_{L}-J_{D}\right.\right.$, where $J_{L}$ and $J_{D}$ are the current densities under illumination and dark condition, respectively, and $V_{\text {eff }}=V_{0}-V$, where $V_{0}$ is the voltage when $J_{\text {ph }}$ $=0$ and $V$ is the applied bias) $\cdot{ }^{[16]}$ As can be seen in Figure $3 c$, $J_{\text {ph }}$ was saturated as $V_{\text {eff }}$ exceeds $1 \mathrm{~V}$, and thus the value of $G_{\max }$ (Table 2) can be obtained from the equation of $J_{\text {sat }}=q \cdot L \cdot G_{\max }$, where $J_{\text {sat }}$ is the saturation photocurrent density, $q$ is the electronic charge, and $L$ is the thickness of $\mathrm{BHJ}$ layer in the device. The fluorinated polymers, PTADTFBT and PTADTDFBT, show 

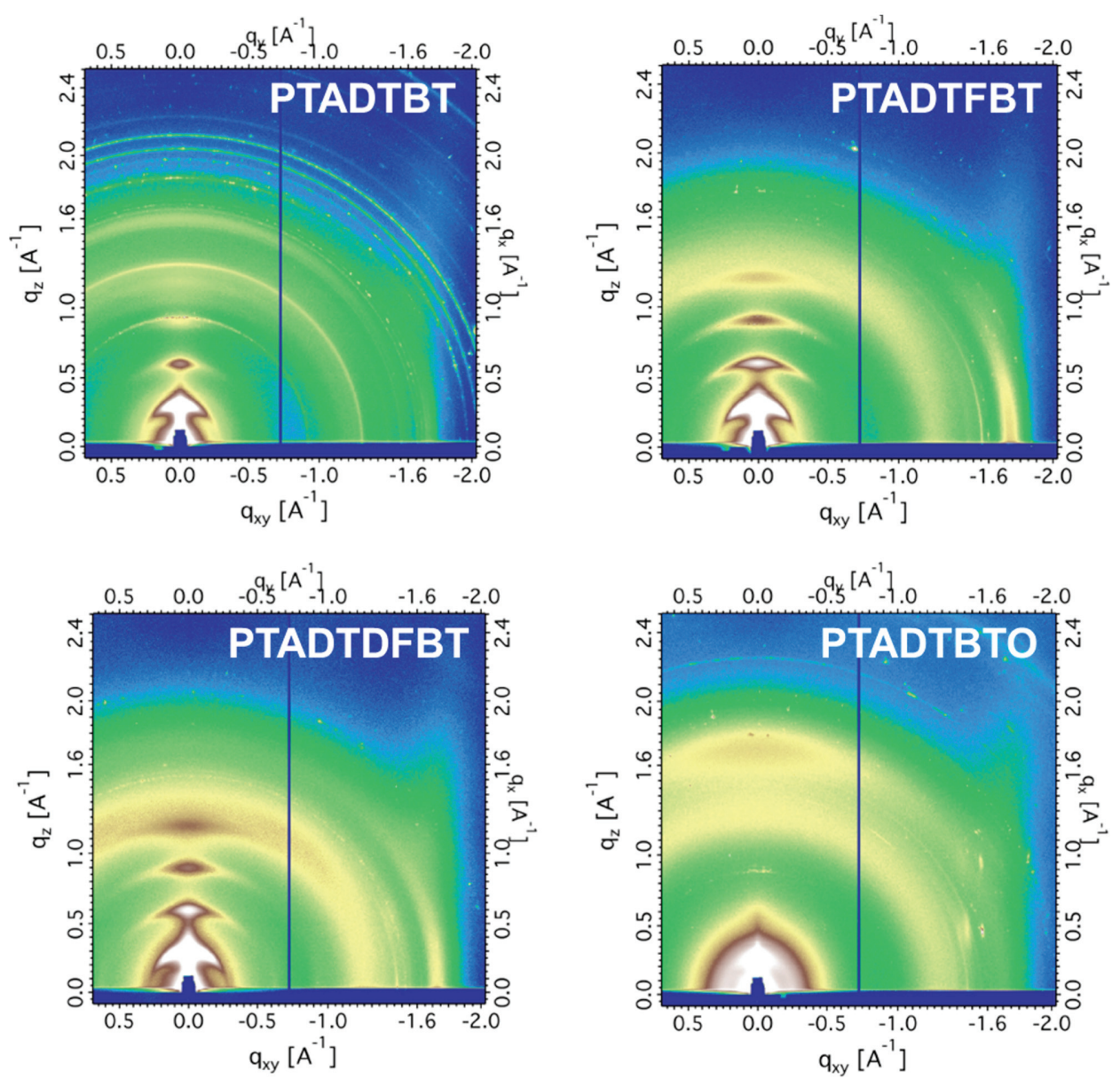

Figure 2. 2D GIWAXS images of polymer thin films.

higher $G_{\max }$ than PTADTBT and PTADTBTO. Higher crystallinity of the fluorinated polymers as compared with nonfluorinated ones (PTADTBT and PTDTBTO) also contributes to higher $J_{\mathrm{SC}} \mathrm{S}$ of fluorinated polymers, because higher crystallinity is expected to afford higher hole transport. It has recently been demonstrated that large dipole change from ground to excited state $\left(\Delta \mu_{\text {ge }}\right)$ facilitates exciton dissociation and thus generation of charge-separated state. ${ }^{[17]}$ When dipole moments between ground and excited states are calculated for corresponding repeating units of four polymers, the fluorinated polymers have larger $\Delta \mu_{\mathrm{ge}}$ than nonfluorinated ones (Table 1), which is well consistent with the incident photon to current density (IPCE) spectra of devices (Figure 3b).

Recombination of charge carriers can be estimated by measuring $J_{\mathrm{SC}}$ as a function of light intensity $\left(P_{\text {light }}\right)$. The dependence of $J_{\mathrm{SC}}$ upon $P_{\text {light }}$ can be represented by a power law equation, $J_{\text {SC }} \propto\left(P_{\text {light }}\right) \alpha$, where $\alpha$ is recombination parameter. ${ }^{[18]}$ Since $\alpha=1$ corresponds to the absence of photocurrent loss from the bimolecular recombination, higher $\alpha$ values for fluorinated polymers $(0.98$ for PTADTDFBT and 0.96 for PTADTFBT) than the values for PTADTBT (0.92) and PTADTBTO (0.92) suggest that the bimolecular recombination is more suppressed in fluorinated polymers than nonfluorinated polymers. In short, the above results lead us to conclude that the enhanced charge generation with reduced charge recombination in the blends of fluorinated polymers and $\mathrm{PC}_{71} \mathrm{BM}$ affords higher $J_{\mathrm{SC}}$.

The morphology of polymer/ $\mathrm{PC}_{71} \mathrm{BM}$ blend was observed by transmission electron microscope (TEM), as shown in Figure 4. It has been well recognized that there is a strong correlation between polymer: $\mathrm{PC}_{71} \mathrm{BM}$ blend morphology and photovoltaic performance. While the blends of PTADTFBT:PC ${ }_{71} \mathrm{BM}$ and PTADTDFBT:PC ${ }_{71} \mathrm{BM}$ exhibit finely phase-separated morphology with fibril-like nanostructure, the PTADTBTO: $\mathrm{PC}_{71} \mathrm{BM}$ blend shows homogeneous-like morphology without distinct phase-separation. Since the standard TEM cannot often detect phase separation in organic photovoltaic (OPV) materials, more advanced TEM techniques could give more detail information on the morphology of $\mathrm{BHJ}$ films. ${ }^{[19]}$ It should be noted here that the formation of fibril nanostructure is closely related to the crystallinity of the polymer: the higher crystallinity, the better formation of fibril nanostructure.

In summary, we designed and synthesized medium bandgap polymers $\left(E_{\mathrm{g}}=1.8-2.1 \mathrm{eV}\right)$ based on TA and BT derivatives with different substituents, as D and A units, respectively. The optical, electrochemical, intermolecular, and charge transport characteristics were finely tuned by changing the type of substituent. Fluorine-substituted polymers exhibit improved charge generation with reduced bimolecular recombination as compared with 

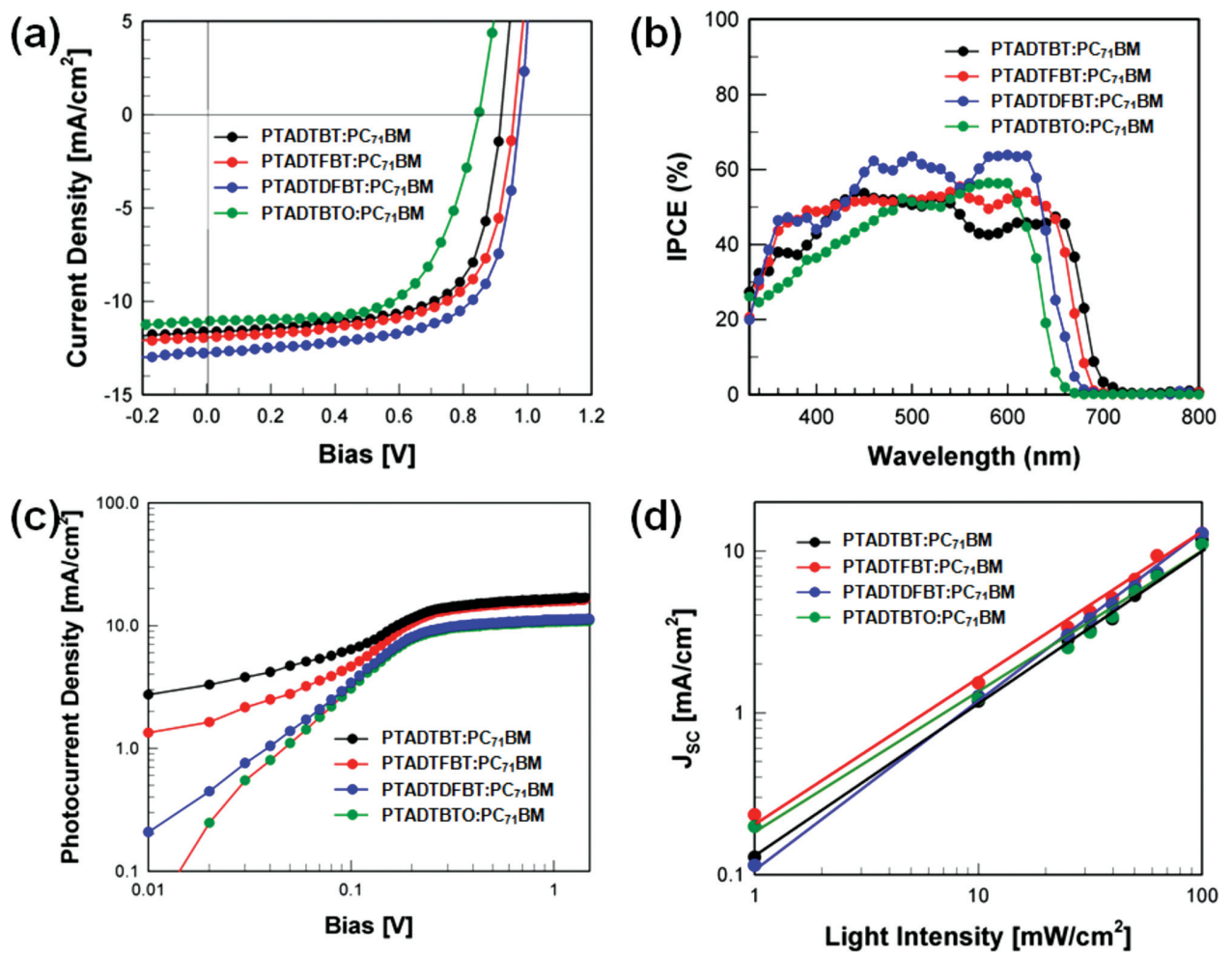

Figure 3. a) $J-V$ curves; b) IPCE spectra; c) the plot of $J_{\text {ph }}$ versus $V_{\text {eff; }}$ and d) correlation of relative $J_{\text {ph }}$-light intensity of the PSC devices based on different polymers.

nonfluorinated or other substituted ones. As a consequence, the di-fluorinated polymer, PTADTDFBT, exhibits a high PCE of $8.05 \%$, which is the highest performance among medium bandgap polymers reported so far. Particularly, the high performance was achieved for the device as fabricated from simple spincoating without addition of additive and posttreatment. This work not only demonstrates that the TA unit is a promising building block for synthesis of medium bandgap polymer for high performance single junction PSCs but also suggests that the fluorine atom substitution on conjugated polymer can tune its frontier energy levels and thereby improve the photovoltaic performance.

\section{Experimental Section}

Synthesis of Polymers: Synthesis details of monomers are described in Supporting Information. For synthesis of PTADTBT, a solution of (5, 5'-(9,10-bis (5-(2-hexyldecyl) thiophene-2-yl) anthracene-2,6-diyl) bis(thiophene-5,2-diyl))bis(trimethylstannane) (128.1 mg, $0.1 \mathrm{mmol}$ )

Table 2. Optimized device parameters of the PSCs.

\begin{tabular}{lcccccc}
\hline Polymer & $\begin{array}{c}V_{\mathrm{OC}} \\
{[\mathrm{V}]}\end{array}$ & $\begin{array}{c}J_{\mathrm{SC}} \\
{\left[\mathrm{mA} \mathrm{cm}^{-2}\right]}\end{array}$ & FF & $\begin{array}{c}\text { PCE } \\
{[\%]}\end{array}$ & $\begin{array}{c}G_{\max } \\
{\left[\mathrm{m}^{-3} \mathrm{~s}^{-3}\right]}\end{array}$ & $\alpha$ \\
\hline PTADTBT & 0.92 & 11.61 & 0.65 & $6.92(6.45)$ & $7.21 \times 10^{27}$ & 0.92 \\
PTADTFBT & 0.95 & 11.95 & 0.64 & $7.27(6.92)$ & $7.52 \times 10^{27}$ & 0.96 \\
PTADTDFBT & 0.97 & 12.77 & 0.65 & $8.05(7.73)$ & $7.84 \times 10^{27}$ & 0.98 \\
PTADTBTO & 0.85 & 11.02 & 0.59 & $4.64(4.51)$ & $6.55 \times 10^{27}$ & 0.92 \\
\hline
\end{tabular}

a)Average PCE in bracket (over 12 devices). and 4,7-dibromobenzo[c][1,2,5]thiadiazole $(29.3 \mathrm{mg}, 0.1 \mathrm{mmol})$ in $4 \mathrm{~mL}$ of anhydrous toluene was refluxed for $48 \mathrm{~h}$ under nitrogen, followed by end-capping using 2-bromothiophene and 2-tributyltinthiophene, successively. After cooling down to room temperature, the solution was precipitated in methanol, filtered, and then purified by Soxhlet extraction successively with methanol, acetone, hexane, methylene chloride, and chloroform. Finally, the chloroform fraction was concentrated, and then the concentrate was precipitated in methanol. The dark purple solid was obtained after filtering by PTFE filter and dried overnight in $70 \%$ yield. For PTADTFBT, PTADTDFBT, and PTADTBTO, $0.1 \mathrm{mmol}$ of 4,7-dibromo5-fluorobenzo[c][1,2,5]thiadiazole, 4,7-dibromo-5,6-difluorobenzo[c] $[1,2,5]$ thiadiazole and 4,7-dibromo-5,6-bis (octyloxy)benzo[c][1,2,5] thiadiazole were used instead of 4,7-dibromobenzo[c][1,2,5]thiadiazole. The yields of the polymerizations were $64 \%, 66 \%$, and $46 \%$ for PTADTFBT, PTADTDFBT, PTADTBTO, respectively.

Fabrication and Characterization of Photovoltaic Cells: Photovoltaic cells were fabricated with an inverted structure of ITO/ZnO $(30 \mathrm{~nm}) /$ polymer: $\mathrm{PC} \mathrm{C}_{71} \mathrm{BM}(120 \mathrm{~nm}) / \mathrm{MoO}_{3}(8 \mathrm{~nm}) / \mathrm{Ag}(120 \mathrm{~nm})$. The patterned indium tin oxide (ITO) glass was cleaned in an ultrasonic bath of acetone and isopropanol, and treated in ultraviolet-ozone chamber for $30 \mathrm{~min}$. Then, $\mathrm{ZnO}$ layer was spin-coated onto the ITO-coated glass substrates from a $\mathrm{ZnO}$ precursor solution $\left(\mathrm{C}_{4} \mathrm{H}_{6} \mathrm{O}_{4} \mathrm{Zn} \cdot 2\left(\mathrm{H}_{2} \mathrm{O}\right)\right.$ in $\mathrm{NH}_{2} \mathrm{CH}_{2} \mathrm{CH}_{2} \mathrm{OH}$ and $\mathrm{CH}_{3} \mathrm{OCH}_{2} \mathrm{CH}_{2} \mathrm{OH}$ ). After thermal annealing at $200{ }^{\circ} \mathrm{C}$ for $1 \mathrm{~h}$, the active layer was spin-coated from the polymer blend solution in o-dichlorobenzene. The solid concentration was set to $35 \mathrm{mg} \mathrm{mL}^{-1}$. After spin coating, the film was placed in a closed jar for slow drying of the solvent. Finally, $\mathrm{MoO}_{3}$ and $\mathrm{Ag}$ were thermally evaporated as an anode electrode at a pressure of less than $10^{-7}$ torr. The active area of each device was $4.00 \mathrm{~mm}^{2}$ defined by a shadow mask. The photovoltaic performance was measured under nitrogen atmosphere inside a glovebox. The current density-voltage $(U-V)$ characteristics were measured with a Keithley 4200 source-meter under AM $1.5 \mathrm{G}\left(100 \mathrm{~mW} \mathrm{~cm}{ }^{-2}\right)$ illumination, which was calibrated using a NREL-certified photodiode

The charge-transport characteristics of the polymers were measured by using thin-film transistors (bottom-gate top-contact device geometry). 

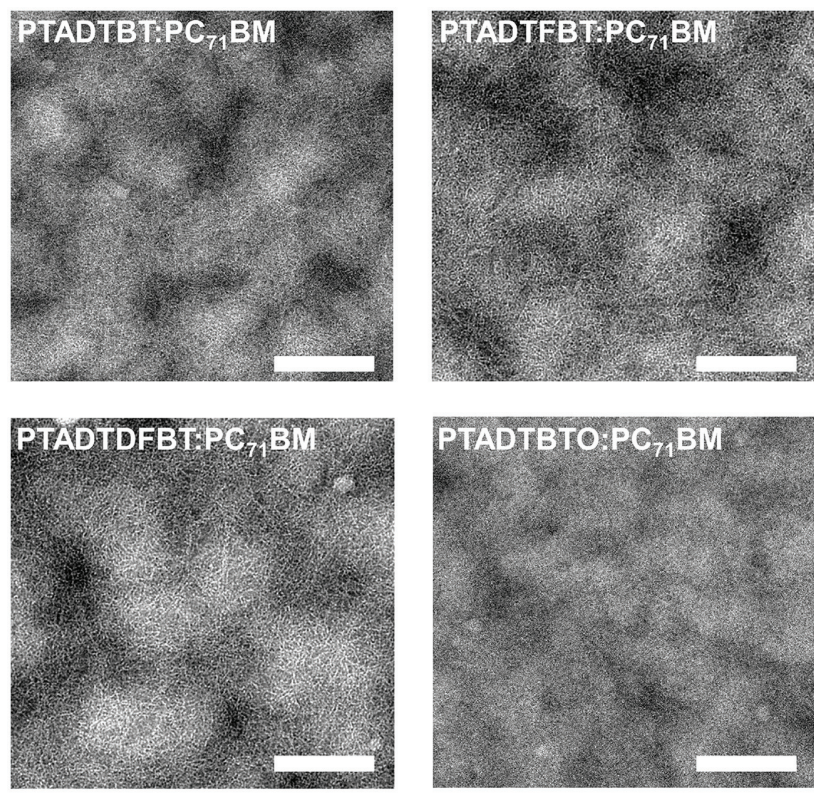

Figure 4. TEM images of blend films made of polymer: $\mathrm{PC}_{71} \mathrm{BM}$ blends. Scale bar is $200 \mathrm{~nm}$.

On the heavily $\mathrm{n}$-doped $\mathrm{SiO}_{2} / \mathrm{Si}$ substrate, the polymers were spin-coated from o-dichlorobenzene solution in thickness of $50 \mathrm{~nm}$. The surface of the substrate was treated by octadecyl trichlorosilane to make hydrophobic dielectric surface. Source and drain electrodes were then thermally evaporated $(60 \mathrm{~nm})$ with channel width and length of 1500 and $30 \mu \mathrm{m}$, respectively. All the devices fabricated in this study were clearly isolated to achieve negligible gate leakage current. All the field effect mobilities were extracted from the saturation regime using the relationship $\mu_{\text {sat }}=\left(2 \cdot I_{D S} \cdot L\right) /\left[W \cdot C\left(V_{G}-V_{\text {th }}\right)^{2}\right]$, where $I_{D S}$ denotes the saturation drain current, $C$ is the capacitance of $\mathrm{SiO}_{2}$ dielectric, $V_{\mathrm{G}}$ is the gate bias, and $V_{\text {th }}$ is the threshold voltage. The SCLC hole mobilities in the active layer of $\mathrm{BHJ}$ were measured in the device configuration of ITO/PEDOT:PSS/ polymer: $\mathrm{PC}_{71} \mathrm{BM} / \mathrm{MoO}_{3} / \mathrm{Au}$. The photovoltaic properties were measured in air using 4200-SCS semiconductor characterization system.

\section{Supporting Information}

Supporting Information is available from the Wiley Online Library or from the author.

\section{Acknowledgements}

The authors thank the Ministry of Education, Korea for financial support through the Global Research Laboratory (GRL) program. T.P.R. and F.L. also acknowledge the support of the US Department of Energy through the Energy Frontier Research Center.

Received: January 9, 2015

Revised: February 27, 2015

Published online: April 10, 2015

[1] a) B. C. Thompson, J. M. J. Fréchet, Angew. Chem. Int. Ed. 2008, 47, 58; b) C. L. Chochos, S. A. Choulis, Prog. Polym. Sci. 2011, 36, 1326; c) J. W. Jung, J. W. Jo, W. H. Jo, Adv. Mater. 2011, 23, 1782; d) C. E. Small, S. Chen, J. Subbiah, C. M. Amb, S.-W. Tsang,
T.-H. Lai, J. R. Reynolds, F. So, Nat. Photonics 2012, 6, 115; e) J. You, L. Dou, K. Yoshimura, T. Kato, K. Ohya, T. Moriarty, K. Emery, C.-C. Chen, J. Gao, G. Li, Y. Yang, Nat. Commun. 2013, 4, 1446.

[2] a) J.-D. Chen, C. Cui, Y.-Q. Li, L. Zhou, Q.-D. Ou, C. Li, Y. Li, Ji.-X. Tang, Adv. Mater. 2015, 27, 1035; b) Y. Liu, J. Zhao, Z. Li, C. Mu, W. Ma, H. Hu, K. Jiang, H. Lin, H. Ade, H. Yan, Nat. Commun. 2014, 5, 5293; c) S.-H. Liao, H.-J. Jhuo, P.-N. Yeh, Y.-S. Cheng, Y.-L. Li, Y.-H. Lee, S. Sharma, S.-A. Chen, Sci. Rep. 2014, 4, 6813.

[3] a) C. J. Brabec, S. Gowrisanker, J. J. M. Halls, D. Laird, S. Jia, S. P. Williams, Adv. Mater. 2010, 22, 3839; b) J. W. Jung, F. Liu, T. P. Russell, W. H. Jo, Energy Environ. Sci. 2012, 5, 6857; c) J. W. Jo, S. S. Kim, W. H. Jo, Org. Electron. 2012, 13, 1322; d) H. X. Zhou, L. Q. Yang, S. C. Price, K. J. Knight, W. You, Angew. Chem. Int. Ed. 2010, 49, 7992; e) I. Osaka, M. Shimawaki, H. Mori, I. Doi, E. Miyazaki, T. Koganezawa, K. Takimiya, J. Am. Chem. Soc. 2012, 134, 3498; f) J.-F. Jheng, Y.-Y. Lai, J.-S. Wu, Y.-H. Chao, C.-L. Wang, C.-S. Hsu, Adv. Mater. 2013, 25, 2445; g) R. S. Ashraf, B. C. Schroeder, H. A. Bronstein, Z. Huang, S. Thomas, R. J. Kline, C. J. Brabec, P. Rannou, T. D. Anthopoulos, J. R. Durrant, I. McCulloch, Adv. Mater. 2013, 25, 2029.

[4] J. W. Jung, F. Liu, T. P. Russell, W. H. Jo, Energy Environ. Sci. 2013, 6, 3301.

[5] P.-L. T. Boudreault, A. Najari, M. Leclerc, Chem. Mater. 2011, 23, 456.

[6] S. C. Price, A. C. Stuart, L. Yang, H. Zhou, W. You, J. Am. Chem. Soc. 2011, 133, 4625.

[7] Y. Dong, X. Hu, C. Duan, P. Liu, S. Liu, L. Lan, D. Chen, L. Ying, S. Su, X. Gong, F. Huang, Y. Cao, Adv. Mater. 2013, 25, 3683.

[8] T. Xu, L. Yu, Mater. Today 2014, 17, 11.

[9] a) P.-L. T. Boudreault, A. Najari, M. Leclerc, Chem. Mater. 2011, 23, 456; b) H.-C. Chen, Y.-H. Chen, C.-C. Liu, Y.-C. Chien, S.-W. Chou, P.-T. Chou, Chem. Mater. 2012, 24, 4766.

[10] a) F. He, W. Wang, W. Chen, T. Xu, S. B. Darling, J. Strzalka, Y. Liu, L. Yu, J. Am. Chem. Soc. 2011, 133, 3284; b) J. B. Lee, K. H. Kim, C. S. Hong and D. H. Choi, J. Polym. Sci., Part A: Polym. Chem. 2012, 50, 2809; c) M. S. Almeataq, H. Yi, S. Al-Faifi, A. A. B. Alghamdi, A. Iraqi, N. W. Scarratt, T. Wang and D. G. Lidzey, Chem. Commun. 2013, 49, 2252.

[11] X. Gong, C. Li, Z. Lu, G. Li, Q. Mei, T. Fang, Z. Bo, Macromol. Rapid Commun. 2013, 34, 1163.

[12] a) M. S. Almeataq, H. Yi, S. Al-Faifi, A. A. B. Alghamdi, A. Iraqi, N. W. Scarratt, T. Wang, D. G. Lidzey, Chem. Commun. 2013, 49, 225; b) C. C. Liu, W. Z. Cai, X. Guan, C. H. Duan, Q. F. Xue, L. Ying, F. Huang, Y. Cao, Polym. Chem. 2013, 4, 3949.

[13] a) I. Benjamin, E. Z. Faraggi, Y. Avny, D. Davidov, R. Neumann, Chem. Mater. 1996, 8, 352; b) G. M. Farinola, A. Cardone, F. Babudri, C. Martinelli, F. Naso, G. Bruno, Maria Losurdo, Materials 2010, 3, 3077; c) H. J. Son, W. Wang, T. Xu, Y. Liang, Y. Wu, G. Li, L. Yu, J. Am. Chem. Soc. 2011, 133, 1885.

[14] a) R. Osterbacka, C. P. An, X. M. Jiang, Z. V. Vardeny, Science 2000, 287, 839; b) J. W. Jung, W. H. Jo, Adv. Funct. Mater. 2010, 20, 2355.

[15] a) J. Kirkpatrick, C. B. Nielsen, W. Zhang, H. Bronstein, R. S. Ashraf, M. Heeney, I. McCulloch, Adv. Energy Mater. 2012, 2, 260; b) S. B. Darling, M. Sternberg, J. Phys. Chem. B 2009, 113, 6215.

[16] J.-L. Wu, F.-C. Chen, Y.-S. Hsiao, F.-C. Chien, P. Chen, C.-H. Kuo, M. H. Huang, C.-S. Hsu, ACS Nano 2011, 5, 959.

[17] J. W. Jo, S. Bae, F. Liu, T. P. Russell, W. H. Jo, Adv. Funct. Mater. 2015, 25, 120.

[18] a) A. C. Stuart, J. R. Tumbleston, H. X. Zhou, W. T. Li, S. B. Liu, H. Ade, W. You, J. Am. Chem. Soc. 2013, 135, 1806; b) S. R. Cowan, J. Wang, J. Yi, Y. J. Lee, D. C. Olson, J. W. P. Hsu, J. Appl. Phys. 2013, 113, 154504.

[19] a) W. Chen, M. P. Nikiforov, S. B. Darling, Energy Environ. Sci. 2012, 5, 8045; b) J. Wen, D. J. Miller, W. Chen, T. Xu, L. Yu, S. B. Darling, N. J. Zaluzec, Microsc. Microanal. 2014, 20, 1507. 